\title{
European VLBI Network dual frequency observations of CSS-GPS candidates
}

\author{
D. Dallacasa ${ }^{1}$, M. Bondi ${ }^{1}$, W. Alef ${ }^{2}$, and F. Mantovani ${ }^{1}$ \\ 1 Istituto di Radioastronomia - CNR, via Gobetti 101, I-40129, Bologna, Italy \\ 2 Max-Planck-Institut für Radioastronomie, Auf dem Hügel 69, D-53121, Bonn, Germany
}

Received August 14; accepted September 12, 1997

\begin{abstract}
We present the results of $S / X(2.3 / 8.3 \mathrm{GHz})$ VLBI snapshot observations of 28 sources drawn from a list of Compact Steep-Spectrum (CSS) and GHz-Peaked Spectrum (GPS) candidates by Dallacasa \& Stanghellini (1991) with additional sources from Barthel et al. (1988) and from Wills (1979). The experiment was carried out with 4 stations of the European VLBI Network (EVN) with the addition of two geodetic antennas. Typical resolutions were about $30 \times 12$ mas and $9 \times 3.5$ mas at $S$ and $X$ bands respectively. Only one source was not detected. Due to the limited $u v$-coverage of the data, only simple structures could be imaged.
\end{abstract}

Key words: galaxies: active; galaxies: jets; galaxies: nuclei; (galaxies:) quasars: general; radio continuum: galaxies

\section{Introduction}

Compact Steep-Spectrum (CSS) and GHz-Peaked Spectrum (GPS) radio sources are intrinsically small objects (Fanti et al. 1990) with high frequency steep spectra $\left(\alpha>0.5\right.$ with $\left.S \propto \nu^{-\alpha}\right)$, projected linear size $<15 \mathrm{kpc}$ and very low linear polarization with a few outstanding exceptions. High frequency radio flux density variability is absent among CSS-GPS galaxies, while it might play a role for quasars, although prominent outbursts have never been observed (Stanghellini et al. 1997).

These properties have been interpreted in terms of youth (Phillips \& Mutel 1982; Carvalho 1985; Mutel \& Phillips 1988; Fanti et al. 1995; Readhead et al. 1996; O'Dea \& Baum 1997), where the jets of a recently triggered radio activity are still digging their way into

Send offprint requests to: D. Dallacasa, Bologna, e-mail: ddallaca@terra.bo.cnr.it the interstellar medium. In this scenario, GPS and CSS sources represent an early-intermediate stage of the radio source life not yet developed into the classical extended double FRII sources (following the nomenclature by Readhead et al. 1996, the Compact Symmetric Objects (CSO) often found among GPS galaxies will evolve into Medium Symmetric Objects (MSO) common among CSS objects, and finally into extended FRII radio galaxies, as the source grows and gets older, diminishing its luminosity by about one order of magnitude).

Alternatively, they could be "frustrated" sources, with anomalously dense nuclear environments impeding the source expansion (van Breugel et al. 1984). Relic extended radio emission is found in about $1 / 5$ of GPS radio sources (Baum et al. 1990; Stanghellini et al. 1990). Baum et al. (1990) interpret this as evidence for smothering produced by infalling gas and dust; this is consistent with the observation of "disturbed" optical images, indication of possible merger activity, often seen in the GPS source hosts (Stanghellini et al. 1993; O'Dea et al. 1996), and with the presence of relic radio emission seen in about $10-20 \%$ of GPS sources (Stanghellini et al. in preparation). An outcome from this scenario is that the radio emitting electrons are confined to the nuclear region by the increased ambient density induced by the merger process.

GPS and CSS sources represent a significant fraction of flux limited catalogues $(15-30 \%$, depending on the selection frequency where the higher fraction is found at higher frequency). They are found at moderate or high redshifts (generally $z>0.2$ but many objects have $z>1.5$ ) and it is remarkable that there does not exist a counterpart of such powerful and compact radio sources in the nearby objects population.

The radio spectra of these objects show a turnover occurring at tens or a few hundreds of $\mathrm{MHz}$ (CSS) or at higher frequencies (GPS) and this phenomenon is generally interpreted in terms of synchrotron self-absorption. 
A direct implication is that generally GPS are smaller than CSS sources.

The samples studied so far (Spencer et al. 1989; Fanti et al. 1990; O'Dea et al. 1990; Dallacasa et al. 1995; Stanghellini et al. 1997) were limited to the most powerful objects, drawn from catalogues at various frequencies. Here we present new result on sources having radio power comparable to the objects in the forementioned papers, but found from other works, not specifically related to the CSS/GPS field.

Furthermore, objects with lower power will help in understanding the relation between the CSS-GPS objects and the other classes of radio sources and possibly with the much weaker nearby CSS-GPS's. Work in this direction is carried out by Snellen et al. (1995)

Section 2 describes the VLBI observations and the data reduction, Sect. 3 briefly presents the sources observed here; in Sect. 4, each source is analyzed, and Sect. 5 summarizes the results.

In this paper we use $H_{0}=100 \mathrm{~km} \mathrm{~s}^{-1} \mathrm{Mpc}^{-1}$ and $q_{0}=0.5$.

\section{Observations and data reduction}

Observations were carried out in two separate VLBI sessions (September 1993 and May 1994) using the 6 antennas listed in Table 1 . The data (taken in right circular polarization, $\mathrm{RCP}$ ) were recorded in MK3 mode A, allowing a total bandwidth of $56 \mathrm{MHz}$. The fourteen $4-\mathrm{MHz}$ bands were split between $X$ band $(8.3 \mathrm{GHz})$ with 8 channels $(32 \mathrm{MHz})$ and the $S$ band with 6 channels $(24 \mathrm{MHz})$. Due to hardware limitations, Noto could record only 4 channels at $X$ band in session 1 , and again 4 at $X$ band and 2 at $S$ band in session 2 .

Table 1. Telescope participating in the observations. The sensitivity given in the table is the ratio between the system temperature (in $\mathrm{K}$ ) and the gain (in $\mathrm{K} / \mathrm{Jy}$ ). Low values mean good sensitivities

\begin{tabular}{lrrr}
\hline \hline Antenna & $\begin{array}{r}\text { Diam. } \\
(\mathrm{m})\end{array}$ & $\begin{array}{r}\text { Sens. (Jy) } \\
X \text {-band }\end{array}$ & $\begin{array}{r}\text { Sens. (Jy) } \\
S \text {-band }\end{array}$ \\
\hline Medicina (I) & 32 & 240 & 850 \\
Onsala (S) & 20 & 2070 & 1530 \\
Effelsberg (D) & 100 & 40 & 250 \\
Noto (I) & 32 & 1800 & 1450 \\
Wettzell (D) & 22 & 660 & 880 \\
Matera (I) & 22 & 3050 & 3330 \\
\hline \hline
\end{tabular}

The VLBI data were correlated at the MPIfR VLBI MK3 correlator in Bonn. A-priori calibration, fringe fitting, editing, self-calibration and imaging were then performed by means of the Astronomical Image
Processing System (AIPS) developed at the National Radio Astronomy Observatory (NRAO).

For unknown reasons, at $X$-band the sensitivity of Onsala in session 1 was about a factor of 100 lower than expected and all the baselines to this station had to be discarded.

Amplitudes were calibrated using 0235+164 (session 1) and $1739+522$ (session 2), both assumed to be unresolved on our baselines, and with flux densities measured at Effelsberg during the VLBI run. The a-priori amplitude calibration suffered from the lack of the TSYS measurements at Wettzell, while at other sites narrow-band and time-varying radio interferences at $S$ band corrupted the accuracy of the TSYS reported in the observing logs, and reduced the correlated amplitude due to occasional saturation of the detector. The final effect is a reduction in the accuracy of the absolute flux density calibration and in a reduced dynamic range of the images.

Each source was observed for a number of 13-min scans (3-5, depending on the source declination in session 1 and 8-10 in session 2) in a range of hour angle, in order to allow the imaging of simple morphologies.

In session 1 we also observed 1607+268 (CTD 93, with a well known double structure) in order to test the reliability of our results.

\section{The sample}

The sources presented here are drawn from a working list of CSS-GPS radio sources and candidates (Dallacasa \& Stanghellini 1991) with the addition of a dozen of CSS quasars from Barthel et al. (1988) and from Wills (1979). They do not represent a complete sample. Most of the objects are high redshift quasars known to be unresolved (or marginally resolved) at arcsecond resolution.

The radio information on these sources is generally sparse, and their radio spectra have been built with a few points from observations spanning many years. This allows the inclusion of variable, flat-spectrum sources, appearing as GPS objects in case the high frequency data were acquired during a low state of radio activity. Therefore the integrated radio spectra presented here can be misinterpreted, particularly for the objects with very few measurements.

\section{Results and comments on individual sources}

In this section we briefly describe the radio structure and the integrated radio spectrum of each source. The spectra in Fig. 1 have been obtained from measurements found in the literature. We used the proper error on the flux density when reported, otherwise we attributed a $5 \%$ formal error to the measurements. Images are presented in Fig. 2 for the sources with significant VLBI structure only, and the components we revealed here are labelled on the images accordingly with the tables described in Sect. 5 . 
Table 2. Observed sources: Col. 1 shows the IAU name, Col. (2) the optical ID, Col. 3 the redshift from literature data, Col. 4 the radio power at $5 \mathrm{GHz}$, Col. 5 displays the largest angular size, Col. 6 contains the VLBI classification from our data ( $\mathrm{P}=$ pointlike; $\mathrm{S}=$ single component, resolved either at $S$ or at $X$ band, or both; $\mathrm{D}=$ double; $\mathrm{C}-\mathrm{J}=$ core-jet; $\mathrm{T}=$ triple; CPLX $=$ complex. ND = Not Detected). Columns 7 and 9 report the total cleaned flux density ad 2.3 and $8.3 \mathrm{GHz}$ respectively, while Cols. 8 and 10 show the resolution achieved in the images at 2.3 and $8.3 \mathrm{GHz}$. Column 11 indicates where the source has been drawn from: DS is for Dallacasa \& Stanghellini (1991), W is for Wills (1979), BMSL is for Barthel et al. (1988). Finally, Col. 12 presents our proposed classification as GPS, CSS, candidate GPS (cG) and flat spectrum source (FS)

\begin{tabular}{|c|c|c|c|c|c|c|c|c|c|c|c|}
\hline $\begin{array}{l}\text { IAU Name } \\
\text { (1) }\end{array}$ & ID & $(3)$ & $\begin{array}{c}\log P \\
\mathrm{~W} \mathrm{~Hz}^{-1} \\
(4)\end{array}$ & $\begin{array}{c}\mathrm{LAS} \\
\operatorname{mas} \\
(5)\end{array}$ & Morph. & $\begin{array}{c}S_{\mathrm{CFD}} \\
\mathrm{Jy} \\
(7)\end{array}$ & $\begin{array}{c}\mathrm{HPBW}_{\mathrm{S}} \\
\text { mas (PA) } \\
(8)\end{array}$ & $\begin{array}{c}X_{\mathrm{CFD}} \\
\mathrm{Jy} \\
(9)\end{array}$ & $\begin{array}{c}\mathrm{HPBW}_{X} \\
\text { mas (PA) } \\
(10)\end{array}$ & $\begin{array}{l}\text { from } \\
(11)\end{array}$ & $\begin{array}{l}\text { class. } \\
(12)\end{array}$ \\
\hline $0201+113$ & Q & 3.61 & 28.4 & & $\mathrm{P}$ & 0.56 & $25.1 \times 10.5(60)$ & 0.67 & $9.7 \times 3.3(53)$ & DS & cG \\
\hline $0237-027$ & Q & 1.116 & 27.3 & & $\mathrm{~S}$ & 0.26 & $20.9 \times 13.7(58)$ & 0.80 & $7.5 \times 4.3(64)$ & W & $\mathrm{cG}$ \\
\hline $0237-233$ & $\mathrm{Q}$ & 2.223 & 28.5 & & $\mathrm{~S} ?$ & 3.27 & $49.7 \times 16.9(37)$ & 1.93 & $21.0 \times 4.5(33)$ & DS & GPS \\
\hline $0500+019$ & G & 0.583 & 26.9 & 10 & $\mathrm{~S}$ & 1.56 & $26.2 \times 11.4(70)$ & 1.36 & $9.8 \times 3.4(68)$ & DS & GPS \\
\hline $0511-220$ & Q & 1.296 & 27.5 & & S?-CJ? & 0.33 & $38.2 \times 19.2(42)$ & 0.86 & $14.7 \times 5.4(49)$ & DS & $\mathrm{cG}$ \\
\hline $0743-006$ & Q & 0.994 & 27.3 & & $\mathrm{P}$ & 0.70 & $26.7 \times 13.0(-78)$ & 1.71 & $8.4 \times 4.5(84)$ & DS & GPS \\
\hline $0922+005$ & Q & 1.72 & 27.6 & & S?-CJ? & 0.45 & $24.4 \times 12.5(-73)$ & 0.41 & $10.0 \times 4.5(-87)$ & $\mathrm{W}$ & FS \\
\hline $0941-080$ & $\mathrm{G}$ & 0.228 & & 50 & $\mathrm{D}$ & 0.92 & $37.9 \times 18.0(-57)$ & 0.20 & $8.2 \times 5.4(-81)$ & DS & CSS \\
\hline $0941+261$ & Q & 2.906 & 27.7 & & ND & ND & - & ND & - & BMSL & CSS \\
\hline $1143-245$ & $\mathrm{Q}$ & 1.950 & 27.9 & & $\mathrm{~S}$ & 1.11 & $40.7 \times 20.3(41)$ & 0.92 & $16.8 \times 6.0(39)$ & $\mathrm{DS}$ & GPS \\
\hline $1237-101$ & Q & 0.753 & 26.9 & & S & 0.82 & $23.4 \times 14.3(51)$ & 0.67 & $7.9 \times 4.2(47)$ & DS & GPS \\
\hline $1317-005$ & Q & 0.892 & 26.8 & & $\mathrm{P}$ & 0.18 & $20.9 \times 13.0(-54)$ & 0.11 & $7.1 \times 3.8(50)$ & $\mathrm{W}$ & CSS \\
\hline $1502+036$ & $\mathrm{Q}$ & 0.411 & 26.3 & & $\mathrm{~S}$ & 0.51 & $28.6 \times 11.3(56)$ & 0.61 & $13.5 \times 3.2(46)$ & $\mathrm{W}$ & FS \\
\hline $1518+047$ & $\mathrm{G}$ & 1.296 & 27.5 & 150 & $\mathrm{D}$ & 1.69 & $22.1 \times 10.2(53)$ & 0.40 & $10.5 \times 3.6(44)$ & $\mathrm{DS}$ & GPS \\
\hline $1607+268$ & $\mathrm{G}$ & 0.473 & 26.7 & 60 & $\mathrm{D}$ & 2.39 & $18.7 \times 9.9(66)$ & 0.90 & $9.1 \times 3.7(48)$ & $\mathrm{DS}$ & GPS \\
\hline $1629+120$ & Q & 1.795 & 27.7 & 1150 & $\mathrm{D}$ & 0.29 & $31.9 \times 10.0(53)$ & 0.11 & $10.0 \times 4.1(44)$ & BMSL & CSS \\
\hline $1848+283$ & Q & 2.56 & 28.1 & & $\mathrm{P}$ & 0.24 & $22.1 \times 10.6(54)$ & 1.44 & $8.3 \times 3.4(51)$ & $\mathrm{DS}$ & GPS \\
\hline $2044-027$ & Q & 0.942 & 27.1 & 40 & $\mathrm{~S}$ & 0.29 & $28.0 \times 17.9(62)$ & 0.03 & $10.0 \times 4.3(50)$ & DS & CSS \\
\hline $2126-158$ & $\mathrm{Q}$ & 3.268 & 28.4 & & $\mathrm{P}$ & 0.56 & $33.6 \times 14.7(42)$ & 0.86 & $13.0 \times 3.9(41)$ & DS & $\mathrm{cG}$ \\
\hline $2128+048$ & $\mathrm{G}$ & 0.99 & 27.5 & 60 & $\mathrm{D} ?$ & 2.25 & $24.1 \times 12.1(61)$ & 1.28 & $8.1 \times 3.7(53)$ & DS & GPS \\
\hline $2137+209$ & $\mathrm{Q}$ & 1.576 & 27.4 & 50 & S & 0.63 & $24.6 \times 9.8(52)$ & 0.23 & $9.6 \times 3.3(46)$ & DS & CSS \\
\hline $2210+016$ & G & $\ldots$ & & 100 & $\mathrm{~T}-\mathrm{CPLX}$ & 1.20 & $23.6 \times 12.3(60)$ & 0.40 & $9.7 \times 3.7(54)$ & $\mathrm{DS}$ & CSS \\
\hline $2223+210$ & Q & 1.959 & 27.9 & & S & 0.79 & $22.3 \times 10.0(57)$ & 1.23 & $8.8 \times 2.9(50)$ & BMSL & CSS \\
\hline $2351-006$ & $\mathrm{Q}$ & 0.46 & 26.0 & & S & 0.29 & $24.2 \times 12.6(60)$ & 0.27 & $8.7 \times 3.9(65)$ & $\mathrm{W}$ & $?$ \\
\hline $1402-012$ & Q & 2.518 & 27.8 & & $\mathrm{P}$ & 0.49 & $19.1 \times 11.8(-83)$ & 0.29 & $5.2 \times 3.3(-84)$ & $\mathrm{W}$ & $\mathrm{cG}$ \\
\hline $1602+576$ & Q & 2.85 & 27.8 & 120 & $\mathrm{D} ?$ & 0.57 & $17.8 \times 10.6(89)$ & 0.34 & $4.1 \times 2.4(90)$ & BMSL & CSS \\
\hline $1629+680$ & Q & 2.475 & 27.7 & 120 & $\mathrm{C}-\mathrm{J}$ & 0.79 & $20.6 \times 11.0(84)$ & 0.20 & $5.2 \times 3.5(70)$ & BMSL & CSS \\
\hline $1801+010$ & $\mathrm{Q}$ & 1.522 & 27.7 & & $\mathrm{P}$ & 1.64 & $21.6 \times 13.6(68)$ & 1.05 & $5.5 \times 3.2(80)$ & BMSL & $c G$ \\
\hline
\end{tabular}

We usually compare the total flux accounted for by either our data or our image to the "average" flux density at the two observing frequencies as derived in the literature, and we use $F_{S}$ and $F_{X}$ to indicate the fraction of flux density in the images at $S$ and $X$ bands respectively. Often we found discrepant measurements of the total flux density, particularly at high frequencies, indication of either large unquoted systematic errors (or confusion) or intrinsic source variability, most effective at $X$ band. The comparison therefore should be taken with care and the fraction of the flux density reported here is only indicative of the possible presence of significant radio emission missed by the VLBI observations. We would like to re- mark that snapshot data taken with a limited number of antennas like in our case are likely to miss complex radio emitting regions with angular sizes larger than a few tens of milliarcsecond.

\subsection{Session 1}

As mentioned in Sect. 2, Noto could not record data at $S$-band, and the visibilities to Onsala were of no use at $X$-band. 

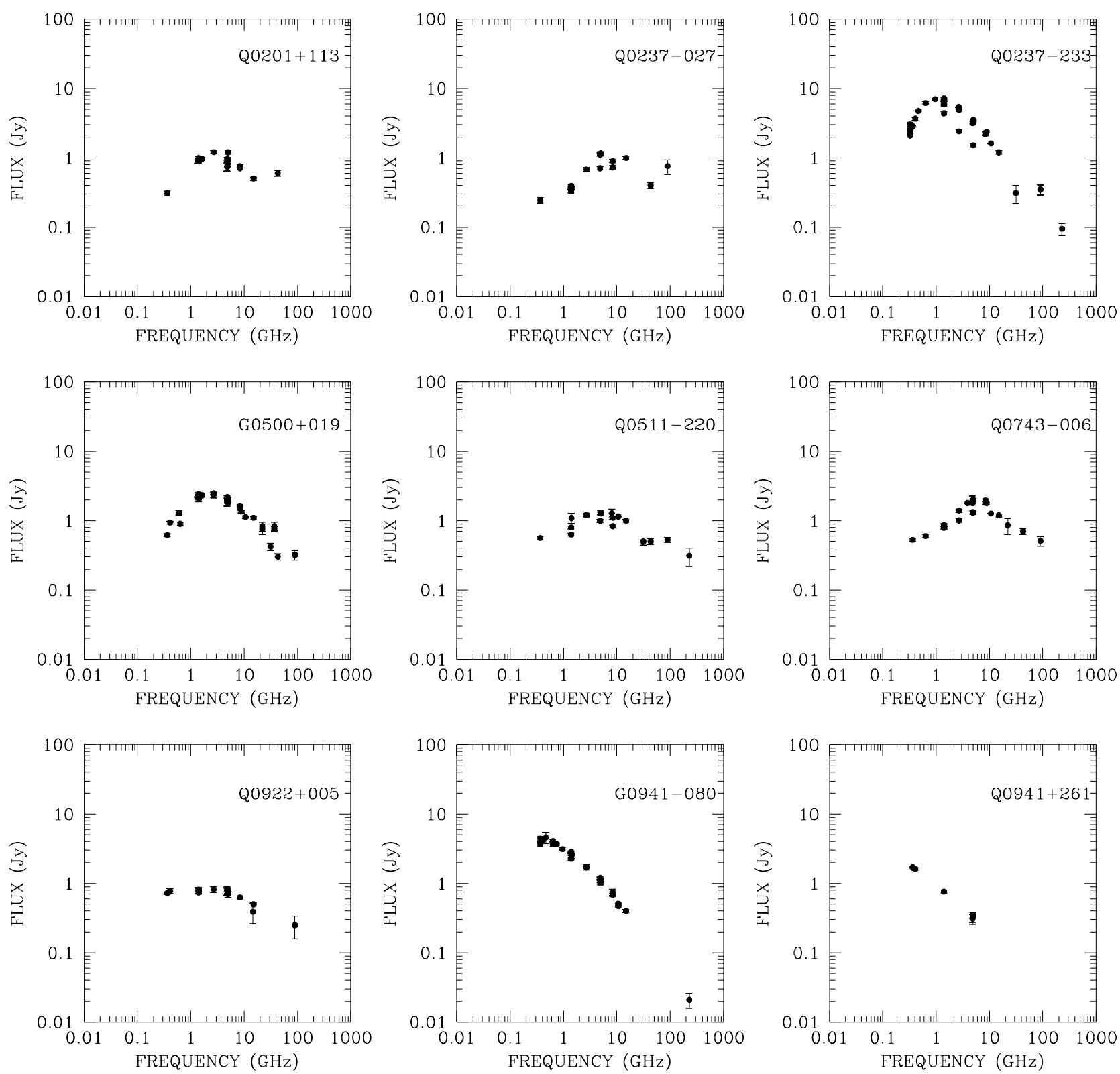

Fig. 1. Radio spectra of the CSS-GPS candidates observed here. The spectral points have been taken from literature

$0201+113[Q, \quad z=3.61]$

Our images at both bands show a single component. The correlated flux density at $X$ band (where the whole flux density is accounted for in our data) is larger than that at $S$-band $\left(F_{S} \sim 50 \%\right)$. Hodges et al. (1984) detected an unresolved component in their VLBI data at $18 \mathrm{~cm}$.

The radio spectrum from nearly simultaneous observations obtained with the Very Large Array (VLA) is presented by O'Dea et al. (1990) and can be considered GPSlike with a peak around $4 \mathrm{GHz}$. However, some variability of the flux density has been observed at $5 \mathrm{GHz}$, and the value of $0.6 \mathrm{Jy}$ at $43 \mathrm{GHz}$ reported in the new VLA calibrators manual suggests a flattening of the spectrum at high frequencies and possibly confirms the variability of the flux density. The optical spectrum shows a damped Ly $\alpha$ absorption feature at $z=3.387$ (White et al. 1993), confirmed by redshifted HI absorption against the quasar detected by de Bruyn et al. (1996).

$0237-027[Q, z=1.116]$

The $X$ band image is dominated by a pointlike component responsible for the whole flux density, while in the $S$ band the source is much weaker and marginally resolved in $\mathrm{PA} \sim 110^{\circ}\left(F_{S} \sim 40 \%\right)$. The spectrum built with data from literature is grossly of GPS type and peaks at 

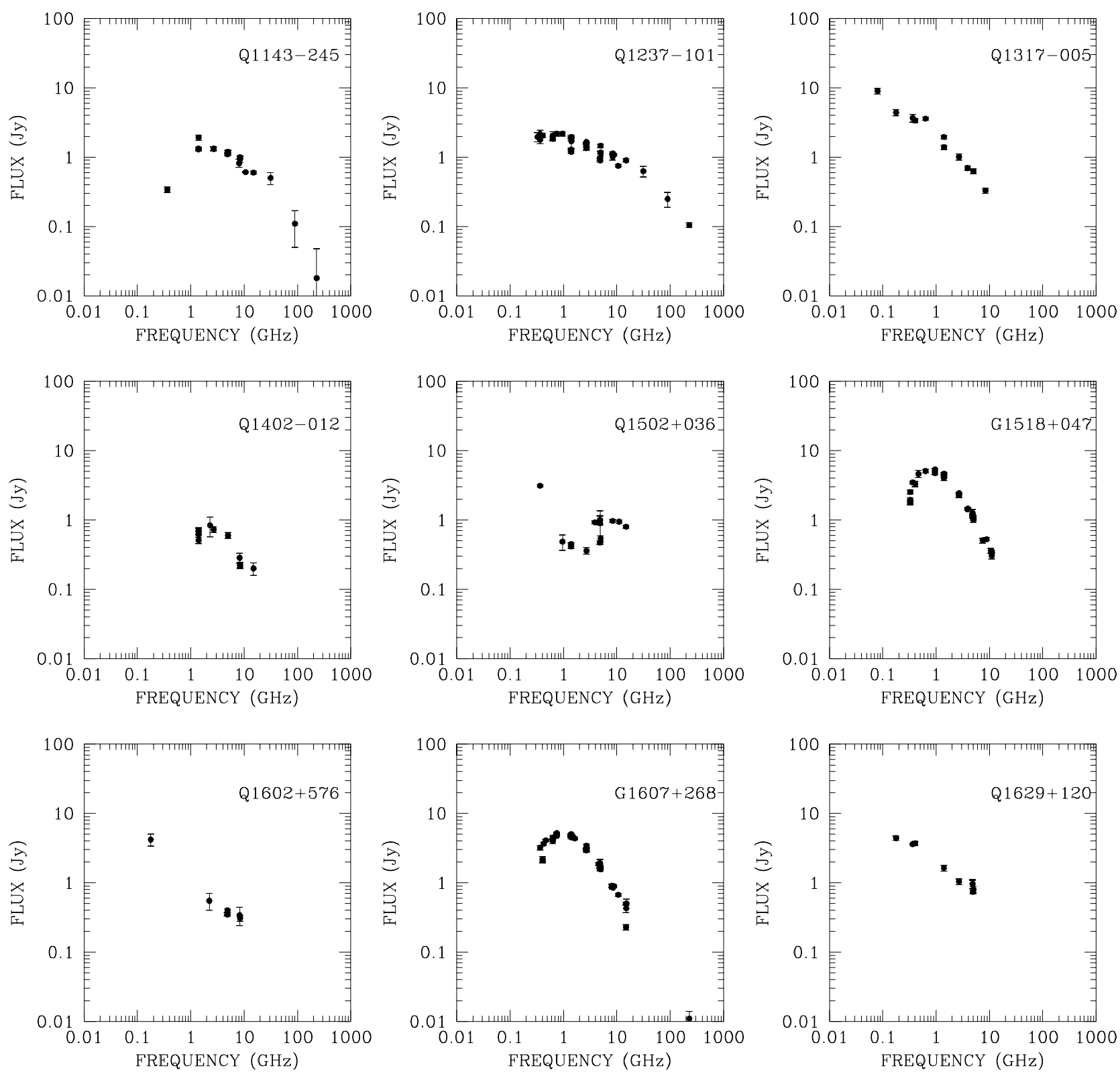

Fig. 1. continued

about $5 \mathrm{GHz}$. However, a recent measurement at $5 \mathrm{GHz}$ by Griffith et al. (1995) revealed a decrease of about $40 \%$ of the flux density with respect to other values previously published (e.g. Perley 1982), and other measurements at high frequencies suggest a possible flattening and/or radio variability of the source. The $90 \mathrm{GHz}$ flux density reported by Steppe et al. (1992) is suggestive of either a rising $\mathrm{mm}$ component or significant variability at $\mathrm{mm}$ wavelengths.

This high redshift quasar was detected in the X-ray by the Einstein satellite (Wilkes et al. 1994).
$0237-233[Q, z=2.223]$

This high redshift quasar has a large number of absorption features in the optical spectrum (see for example Aldcroft et al. 1994). There is also an indication of X-ray variability (Wilkes et al. 1994).

The integrated radio spectrum reported by Kühr et al. (1981), O'Dea et al. (1990) and by Steppe et al. (1995) is typical of a GPS radio source with a spectral peak around $1 \mathrm{GHz}$. However by comparing the forementioned spectra with the flux densities reported in the new Parkes 

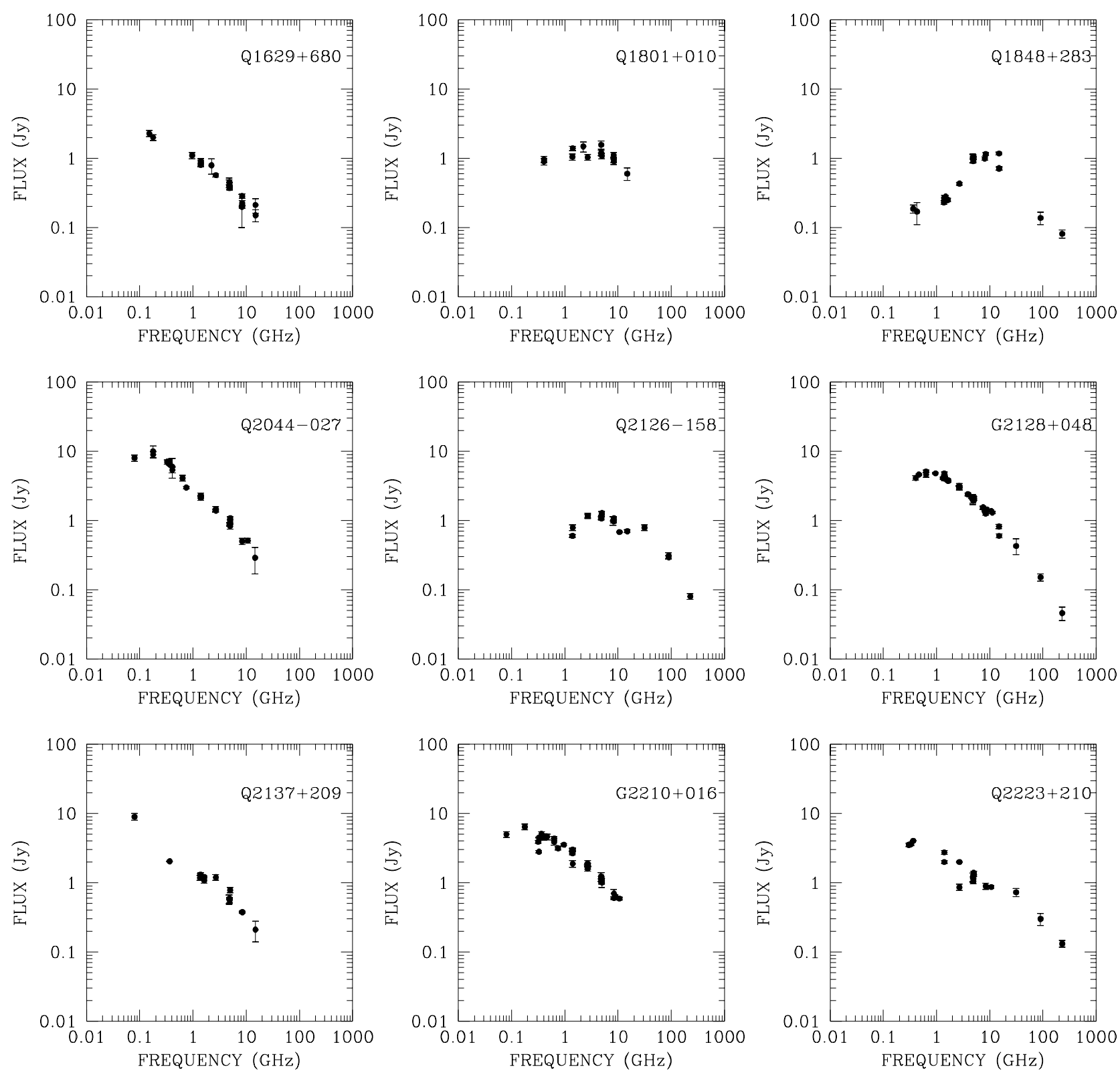

Fig. 1. continued

catalogue, we can infer a significant decrease of the intensity at all the frequencies, although the spectrum still is of GPS type (see Fig. 1). On the contrary, 10 years of monitoring (Waltman et al. 1991) show a nearly constant flux density at both 2695 and $8085 \mathrm{MHz}$. The VLBI image at $X$ band in Fig. 2 shows two very asymmetric components (flux density ratio $12: 1$ ) separated by about 12 mas and accounting for nearly the whole flux density at this frequency. The source is not resolved at $S$ band $\left(F_{S} \sim 65 \%\right)$, due to the lower resolution. The $S$-band model and image presented by Preston et al. (1989) show an asymmetric double source elongated in $\mathrm{PA} \sim 90^{\circ}$ with a separation of about 20 mas between the two components.
$0500+019[G, z=0.583]$

There has been some debate on the optical identification: formerly associated with a quasar by Fugmann \& Meisenheimer (1988), now the radio source host has been classified as a galaxy at $z=0.583$ by de Vries et al. (1995).

The source was not resolved with VLBI by Hodges et al. (1984) at $18 \mathrm{~cm}$. Whilst the structure is barely resolved at $S$ band (with $F_{S} \sim 65 \%$ ), at $X$ band (Fig. 2) the radio emission shows two very asymmetric regions separated by $\sim 10$ mas in $\mathrm{PA} \sim-180^{\circ}$ and accounting for almost the whole flux density. Our data are consistent with a global VLBI image at $5 \mathrm{GHz}$ by 


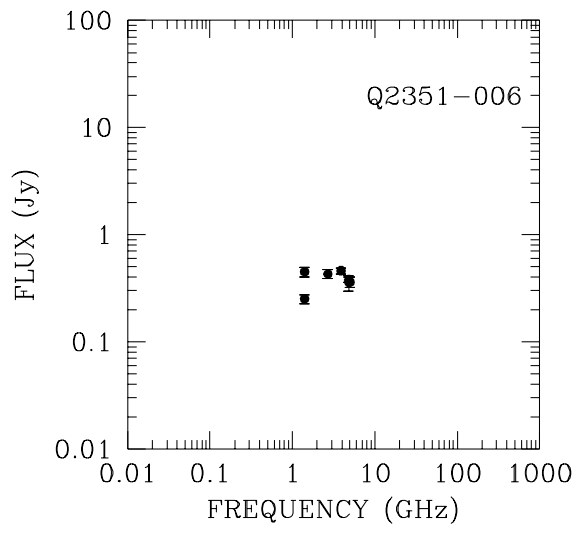

Fig. 1. continued

Stanghellini et al. (1997). The integrated radio spectrum peaks around $3 \mathrm{GHz}$ and is rather flat at $\mathrm{dm}$ wavelengths and is similar to that presented by O'Dea et al. (1990). There is a significant steepening in the high frequency end ( $\alpha \sim 0.9$ between 10.7 and $31 \mathrm{GHz}$ ). Millimeter observations reported by Wiren et al. (1992) are suggestive of an increased flux density at high frequencies, with a possible flattening of the spectrum.

$0511-220[Q, z=1.296]$

This quasar has a GPS type radio spectrum peaking around $5 \mathrm{GHz}$, although the high frequency spectral index is just about 0.5 . Some variability can be inferred at both 1.4 and $8.4 \mathrm{GHz}$, although the amount of data is too limited to draw firm conclusions.

The VLBI structure is resolved at $X$ band (see Fig. 2) in $\mathrm{PA} \sim-40^{\circ}$ and can be either a core-jet or an asymmetric triple $\left(F_{X} \sim 85 \%\right)$. The $S$ band image instead shows unresolved emission with $F_{S} \sim 25 \%$ only, corresponding to about one third of the total flux density detected at $X$-band. Therefore the radio emission detected here has an inverted spectral index. However, the uncertainties in the accounted flux and structures are larger than in other sources since the source declination is rather low.

$0743-006[Q, z=0.994]$

This GPS quasar has not been resolved by the present observations. Within the errors, at $X$ band the whole flux density of the radio source is accounted for in our image, while at $S$ band the fraction decreases to about $75 \%$. Global VLBI data at $5 \mathrm{GHz}$ show a compact component, a few milliarcsec in size (Stanghellini et al. 1997). The ra- dio spectrum has a convex shape with possible variability around the peak occurring between 5 and $10 \mathrm{GHz}$.

$0922+005[Q, z=1.72]$

The radio spectrum of this quasar turned out to be flat between 5 and $0.4 \mathrm{GHz}$ and therefore the turnover cannot be clearly determined. The radio emission is resolved at $S$ band in $\mathrm{PA} \sim 45^{\circ}$. At $X$ band again the source is just resolved in $\mathrm{PA} \sim 30^{\circ}$. This source was not detected at $2.3 \mathrm{MHz}$ in a single baseline experiment by Morabito et al. (1986). Our VLBI images account for $\sim 55 \%$ and $65 \%$ of the total flux density at $S$ and $X$ bands respectively.

$0941-080[G, z=0.228]$

This galaxy (whose redshift has been provided by de Vries and O'Dea, private communication) is well resolved by the present data at both frequencies. The VLBI morphology is that of a compact double (see the images at both $S$ and $X$-band shown in Fig. 2), typical of many CSS-GPS radio galaxies. In our images $F_{S} \sim 55 \%$ and $F_{X} \sim 30 \%$, clear indication of additional radio emission completely resolved out by the present observations, in particular at $X$ band. If we consider the flux densities measured for the two components on our images, the spectral index is 1.0 and 1.4 for the southern and northern components respectively. A proper measurement would require matched $u v$-coverages; we might get spectral indices steeper than the true ones due to the poorer sampling of the short $u v$-spacings at $X$ band. The radio spectrum peaks at about $0.4 \mathrm{GHz}$, and the high frequency spectral index is $\alpha \sim 1.0$ between 5.0 and $10.7 \mathrm{GHz}$ (derived from the literature).

$0941+261[Q, z=2.906]$

This quasar has not been detected by the present observations at either frequency. The VLA image at $5 \mathrm{GHz}$ by Barthel et al. (1988) shows a misaligned triple source. They identify the quasar with the brightest steepspectrum component and therefore suggest the classification as curved-jet source. The integrated radio spectrum has a nearly constant slope between 0.4 and $5 \mathrm{GHz}$, with a spectral index of 0.6 , although more measurements are required for a proper definition of the spectrum itself. The optical spectrum shows the presence of a number of absorption lines spread at various redshifts (see Hewitt \& Burbidge 1989 for references).

$1143-245[Q, z=1.950]$

The radio spectrum is rather flat, although there are clear indications of a steepening at $\mathrm{mm}$ and sub-mm 

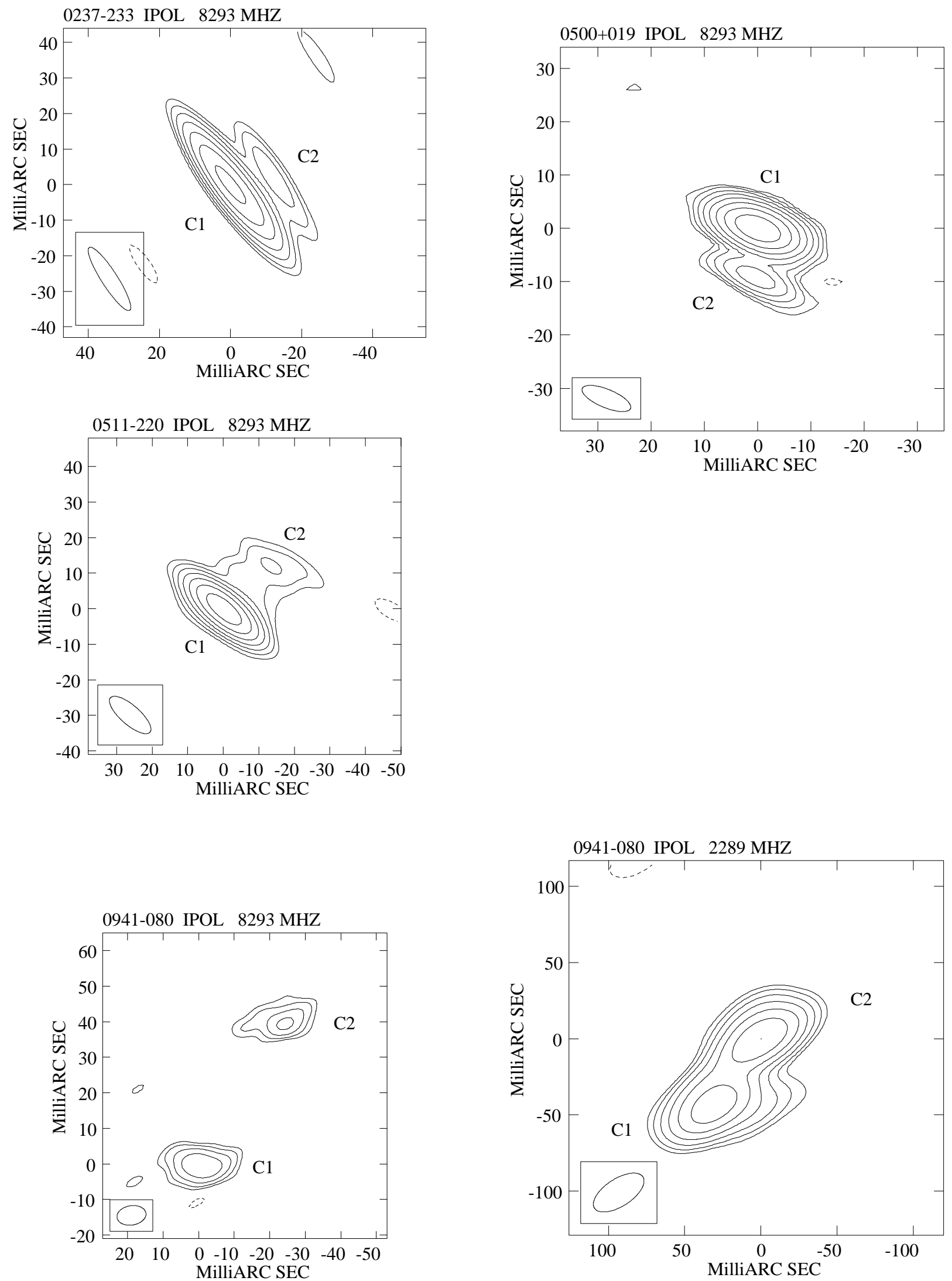

Fig. 2. Images for the sources showing structure resolved in two or more components. The restoring beam for each image is reported in Table 2 . The contour levels are $-1,1,2,4,8,16,32,64,125,250$ times the first contour reported in Tables 3 and 4 for the $X$ and $S$ band images respectively 

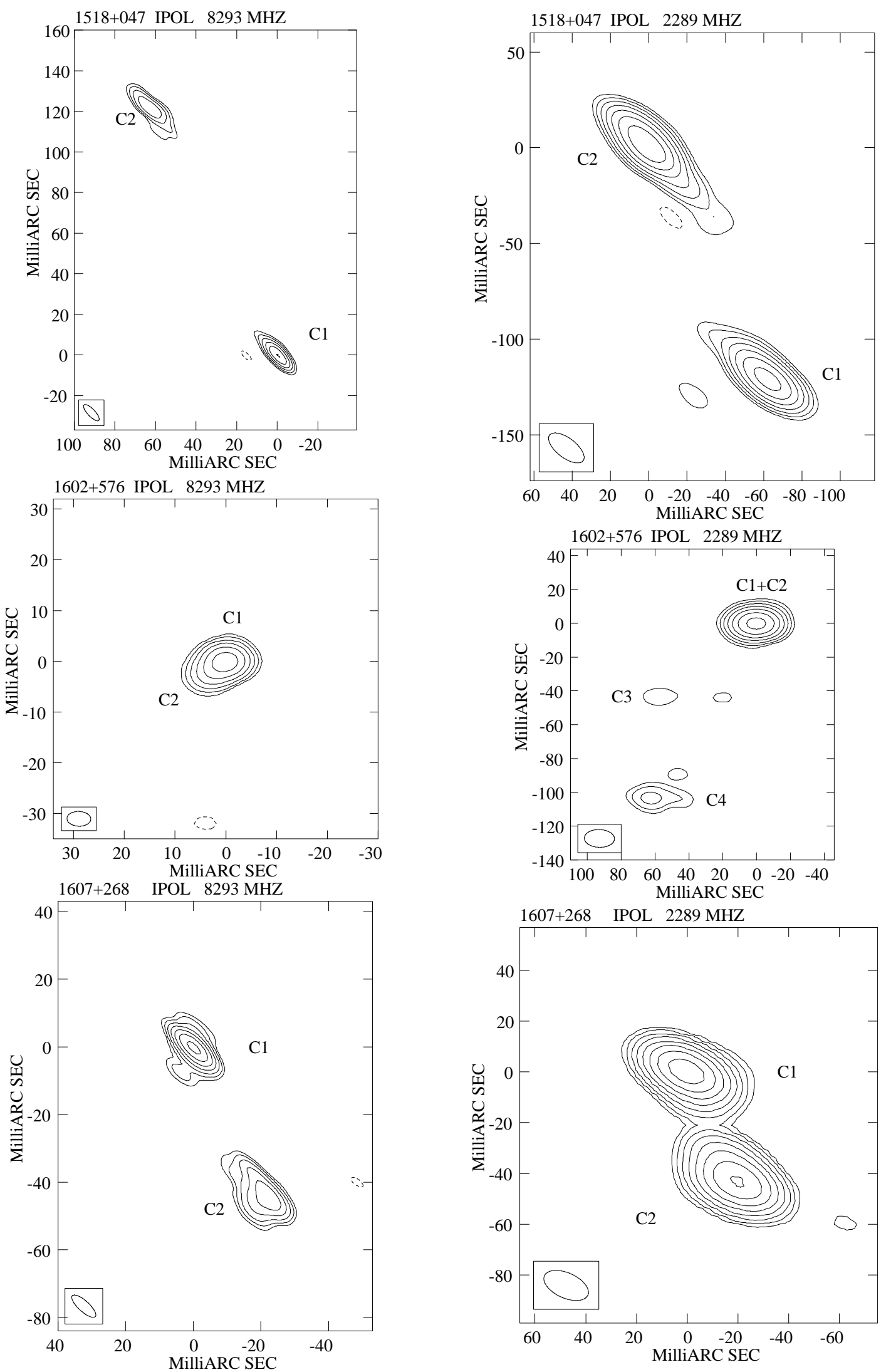

Fig. 2. continued 

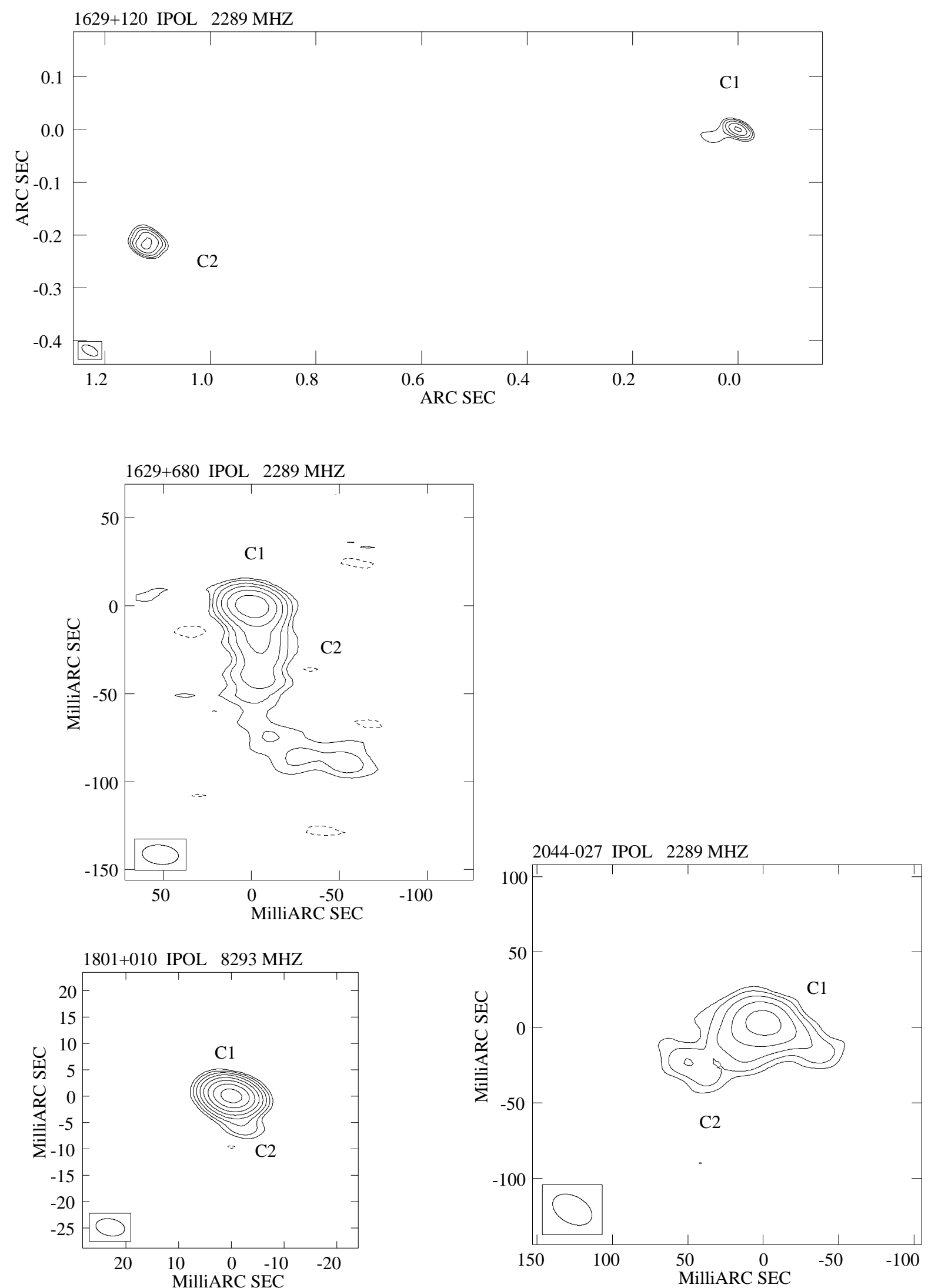

Fig. 2. continued 


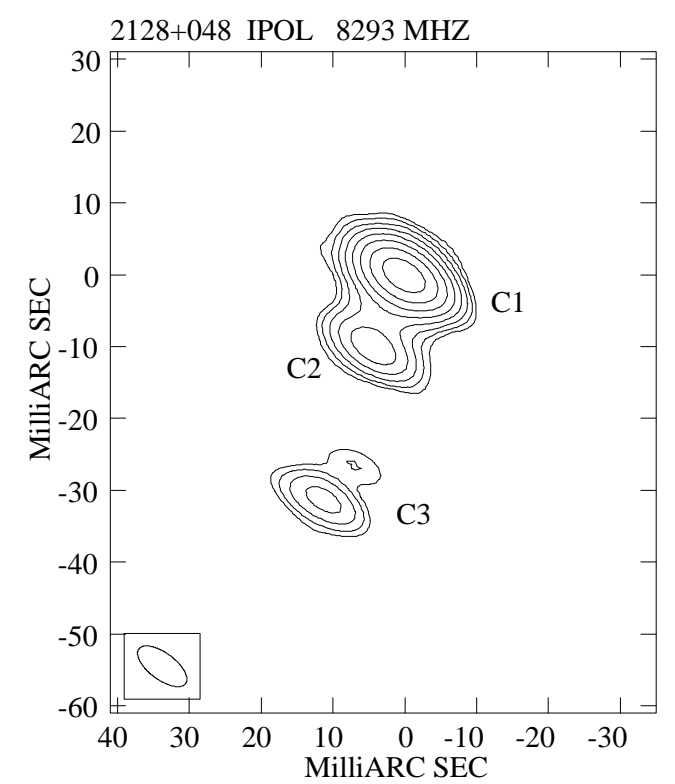

2210+016 IPOL $8293 \mathrm{MHZ}$
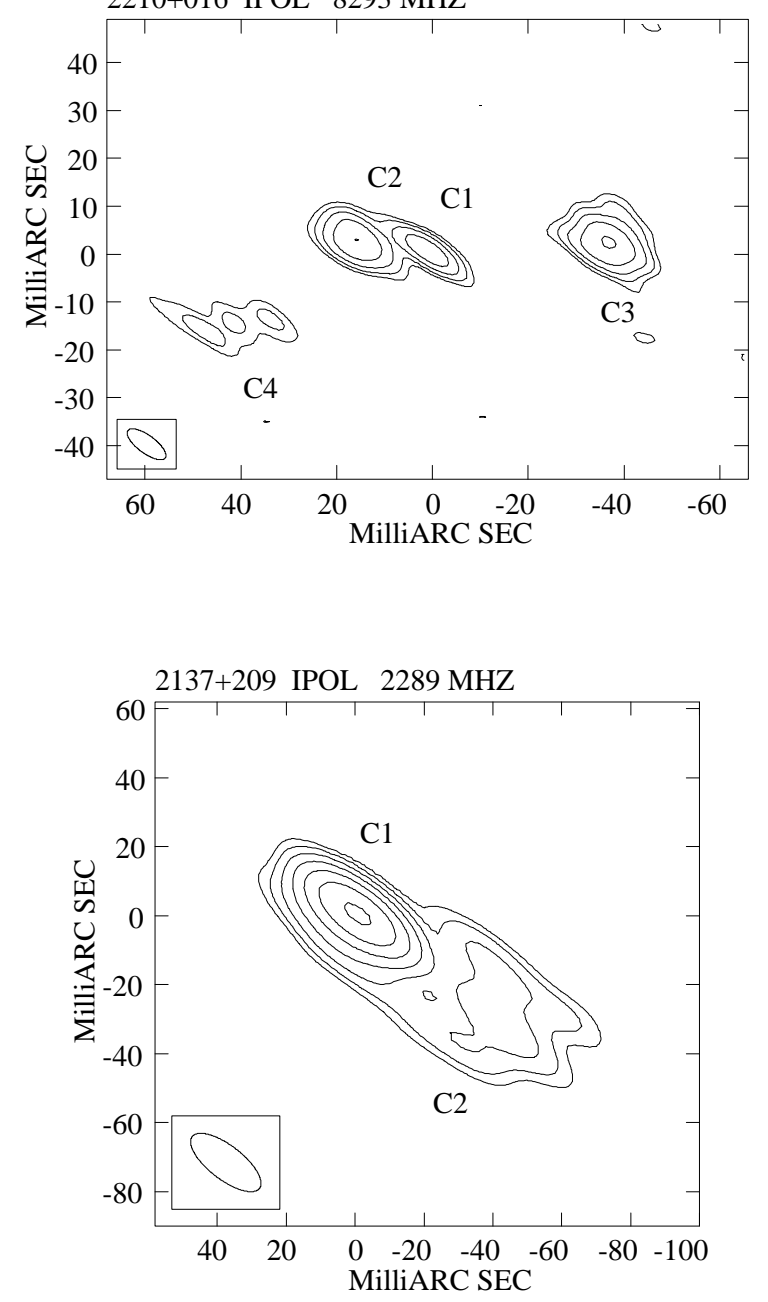
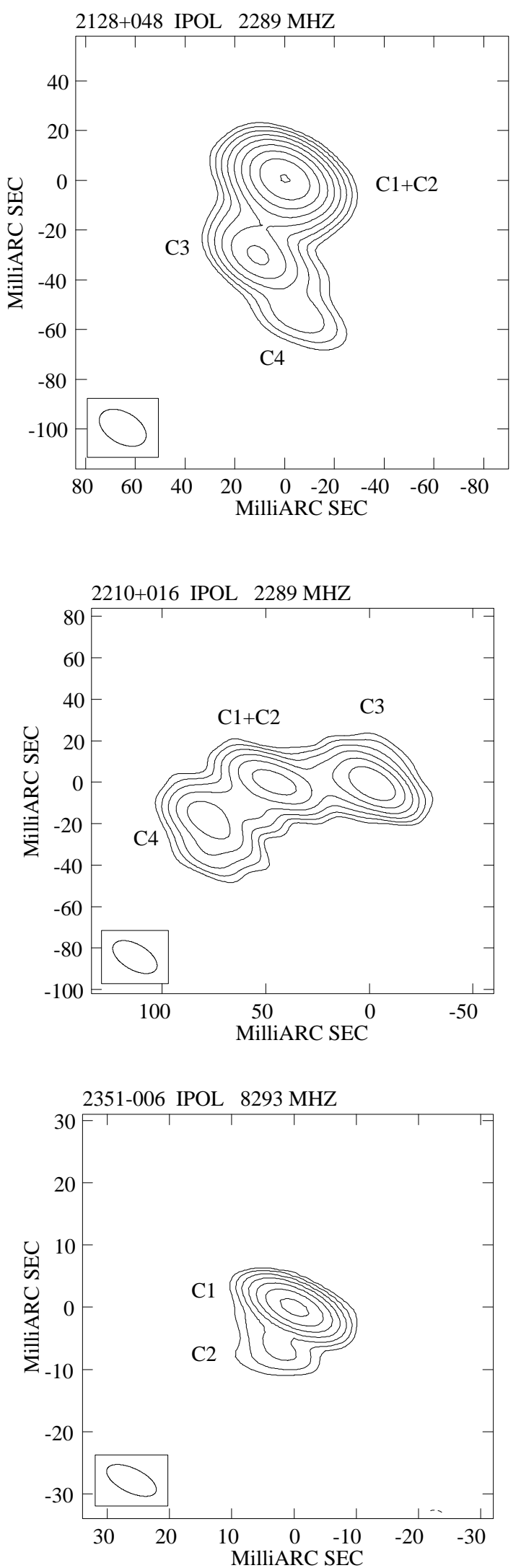

Fig. 2. continued 
wavelengths (Steppe et al. 1992, 1995), and a sharp turnover around $1 \mathrm{GHz}$. The VLBI emission is dominated by a pointlike component, possibly barely resolved at $S$ band, where $F_{S} \sim 85 \%$. At $X$ band the whole flux density of the source is accounted for in our data.

$$
1237-101[Q, z=0.753]
$$

This optically variable quasar has a flat low frequency radio spectrum without a well defined turnover, while there is some progressive steepening at $\mathrm{cm}$ and $\mathrm{mm}$ wavelengths. Some variability can also be inferred from the literature data spanning more than ten years in time. The mas radio emission is dominated by a compact region, barely resolved in $\mathrm{PA} \sim-45^{\circ}$. In our images $F_{X} \sim 65 \%$ and $F_{S} \sim 60 \%$.

This object has also been detected in the X-rays (Wilkes et al. 1994).

$$
1317-005[Q, z=0.892](4 \mathrm{C}-00.50)
$$

The radio spectrum of this quasar is rather steep and does not show any turnover down to $80 \mathrm{MHz}$. The mas structure is dominated by a pointlike component at both frequencies, accounting for a limited fraction of the total flux density ( $\sim 15 \%$ at $S$ and $\sim 25 \%$ at $X$ band); such a straight radio spectrum would imply structures on scales larger than those found by the present observations.

$$
1502+036[Q, z=0.411]
$$

At $\mathrm{cm}$ wavelengths, the radio emission of this object turned out to be variable. At $5 \mathrm{GHz}$ the flux densities reported in the literature differ by a factor of 2 at least. In fact Perley (1982) reports 0.47 Jy while in the Green Bank catalogue its flux density is given as $0.99 \mathrm{Jy}$ and finally the updated flux density in the VLA calibrator manual is $0.90 \mathrm{Jy}$. Moreover, the flux density at $2.7 \mathrm{GHz}$ in the new Parkes Catalogue is only $60 \%$ of the flux density accounted for in our image. Our VLBI images are dominated by a very compact component, marginally resolved at $S$ band, with an inverted spectral index.

$$
1518+047[G, z=1.296](4 \mathrm{C}+04.51)
$$

The radio spectrum of this galaxy peaks at about $0.8 \mathrm{GHz}$ and then sharply declines at lower frequencies. The optically thin spectral index is rather steep, about 1.6. In Fig. 1 we did not plot the measurement at $80 \mathrm{MHz}$ reported in the new Parkes Catalogue, because it is very high (16 Jy, to be compared with the $2 \mathrm{Jy}$ measured at $178 \mathrm{MHz}$ ), possibly affected by confusion, since there is at least another strong source at about 1 arcmin from
$1518+047$ (see the VLA image by Rusk 1988). Some low frequency variability has been detected at $327 \mathrm{MHz}$ by Ghosh et al. (1994).

The mas structure (see the images at both $X$ and $S$ band in Fig. 2) is dominated by two components separated by about 140 mas in $\mathrm{PA} \sim 25^{\circ}$, and they are likely to be the lobes of a medium-size compact symmetric object (MSO, Readhead et al. 1996). The northern region has a steeper spectral index $(\alpha=1.3)$ with respect to the southern $(\alpha=0.9)$, although we must remark that at $X$-band we are likely to have detected the hot-spot region, and a proper comparison would require matched $u v$-coverages. In our images $F_{X} \sim 80 \%$ and $F_{S} \sim 70 \%$. Our result is in basic agreement with Mutel et al. (1985), who presented images at 5 and $1.6 \mathrm{GHz}$, and resolve both components.

$1607+268[G, z=0.473]$

This galaxy, also known as CTD93, has a double VLBI morphology as already presented by Mutel et al. (1985) and various authors afterwards. It belongs to various samples of GPS and CSS sources (Fanti et al. 1990, 1995; O'Dea et al. 1991) and it was observed to test the reliability of the results obtained by the present observations. At both bands (see Fig. 2) the source is dominated by two components with a flux density ratio of about $1: 1.2$, separated by about 48 mas (considering their centroids). The whole flux density is accounted for in the $X$ band image, while $F_{S} \sim 75 \%$. The radio spectrum peaks around $1 \mathrm{GHz}$, and the spectral index of the optically thin region is about 1.4. Moderate flux density variability has been revealed at $327 \mathrm{MHz}$ by Ghosh et al. (1994).

$1629+120[Q, z=1.795](4 \mathrm{C}+12.59)$

The radio spectrum in Fig. 1 is steep $(\alpha=0.7)$; however there are no flux density measurements at frequencies higher than $5 \mathrm{GHz}$, and therefore it is rather difficult to do a proper classification of its shape.

The arcsecond radio emission is that of a triple where the core is identified with the westernmost component of the VLA image by Barthel et al. (1988). Our VLBI image at $S$ band shows two regions separated by $\sim 1.14$ arcsec; at $X$ band only one of them has been detected. Since the westernmost component appears to be unresolved at $S$ band, we tentatively say that component $\mathrm{C} 1$ (see Fig. 2$)$ is the flat spectrum core $(\alpha \sim 0.0$ between our two images). The fraction of the total flux density accounted for in our images is generally small $\left(F_{S} \sim 30 \%\right.$ and we estimate $F_{X} \sim 20 \%$ ). 
$1848+283[Q, \quad z=2.56]$

The radio spectrum of this quasar is peaked around $10 \mathrm{GHz}$, and is characterized by a rather sharp falloff below $5 \mathrm{GHz}$. The radio polarization is also very low if not absent at $\mathrm{cm}$ wavelengths, as found by Rusk (1988). This evidence, together with the VLBI morphology, makes this object a positive GPS candidate. Possible variability can be inferred if we consider the $2 \mathrm{~cm}$ flux density reported by Rusk (1988) which is about 50\% higher than the measurements found on the web page of the VLA calibrators manual and the flux density reported by Owen et al. (1980). Spangler et al. (1983) reported an increase of the high frequencies flux densities on a timescale of about 4 years; the radio spectrum was in both cases of GPS type.

Our images show an unresolved component at both frequencies, and while in the $S$ band image only about $65 \%$ of the total flux is accounted for, in the $X$ band the total flux density in the image exceeds (by about 20\%) the average value derived from the literature, which is further indication of high frequency variability.

$$
2044-027[Q, z=0.942](3 \mathrm{C} 422,4 \mathrm{C}-02.80)
$$

The radio spectrum of this quasar is typical of a CSS source, with a spectral peak of about 10 Jy occurring at about $200 \mathrm{MHz}$. The optical spectrum shows a number of absorption features occurring at a redshift very close to that of the quasar (Aldcroft et al. 1994). The radio emission is resolved with the VLA (see Price et al. 1993) at $6 \mathrm{~cm}$, but it is still dominated by a compact component with $0.5 \mathrm{Jy}$.

Our $S$ band image (Fig. 2) shows well resolved emission with a lobe-like morphology, which accounts for about $\sim 25 \%$ of the total flux density. The fits to the data and and to the image with a single component were rather poor and therefore we decided to fit the two components $\mathrm{C} 1$ and $\mathrm{C} 2$ listed in Table 4 and shown in Fig. 2. In the $X$ band the source is barely detected, and the weak component (probably the hot-spot of the forementioned lobe) contains only about $\sim 5 \%$ of the flux density of the source at this frequency.

$$
2126-158[Q, z=3.268]
$$

This high redshift radio quasar shows a large number of absorption features in the optical spectrum (see Junkkarinen et al. 1991), which has also been found to be very weakly polarized (Wills et al. 1992). The X-ray emission from this quasar is likely to be non variable (Elvis et al. 1994; Bechtold et al. 1994).

The radio spectrum is peaked around $4 \mathrm{GHz}$, although we cannot exclude some variability which can move the intrinsic spectral peak. At arcsecond resolution, Neff \&
Hutchings (1990) find a secondary component with about $5 \%$ of the total flux density at $20 \mathrm{~cm}$, located at about 1.5 arcsec from the core region. They interpret this emission as a bright knot in a jet. Our VLBI images show a pointlike feature at both frequencies, with $F_{X} \sim 90 \%$ and $F_{S} \sim$ $60 \%$. The radio source is resolved at mas resolution by Stanghellini et al. (1997) with a global VLBI observation at $5 \mathrm{GHz}$.

$2128+048\left[\begin{array}{lll}G & z=0.99\end{array}\right]$

The integrated spectrum of this weak galaxy is that typical of a GPS radio source, with a spectral peak occurring at about $600 \mathrm{MHz}$. The VLBI emission is well resolved into a triple ( $X$ band image), with a possible tail detected in the $S$ band only (see Fig. 2). The image at $X$ band accounts for the whole flux density of the source, while the $F_{S} \sim 70 \%$. If we assume that the source core is the central component $\mathrm{C} 2$ of the $X$ band image, this object can be considered a CSO candidate. A global VLBI image at $5 \mathrm{GHz}$ by Stanghellini et al. (1997) tentatively confirms this assumption.

$2137+209[Q, z=1.576]$

This quasar is resolved at both frequencies. In particular at $S$ band (see Fig. 2) the VLBI morphology is rather complex and could be interpreted either in terms of a double or as a lobe with hot-spot. At both frequencies, our images account for about $50 \%$ of the total flux density. It is not easy to describe the integrated radio spectrum due to the scarcity of measurements; there might be some degree of variability at high frequencies as derived from the comparison between the flux density measurements at $6 \mathrm{~cm}$ from the Green Bank Catalogue (Becker et al. 1991) and the new Parkes Catalogue.

$2210+016[G, z$ not available $](4 \mathrm{C}+01.69)$

The optical identification for this source is still controversal. Stickel \& Kühr (1996) find a quasar with $m=21.7$ at $R$-band, while de Vries et al. (1995) find a galaxy with $m=22.0$ at $i$-band. We prefer the latter possibility, according to the VLBI morphology presented here, which is most commonly found among galaxies. The radio spectrum is typical of a CSS source, with a flattening of the slope at a few hundreds MHz. Ghosh \& Rao (1992) find significant flux density variability at $327 \mathrm{MHz}$, while there are no indications of variability at high frequencies.

The VLBI structure revealed in our images is rather complex (Fig. 2). At $S$ band the radio emission is dominated by three components (accounting for about $65 \%$ of the total flux density) which are further resolved into a number of components in the $X$ band image (where 
Table 3. Source models at $X$ band $(8.3 \mathrm{GHz})$. Column 1 gives the source name, Col. 2 shows the component name derived from the images of Fig. 2; Cols. 3 and 4 show the radial distance and position angle of the various component derived with respect to component C1. Column 5 gives the flux density; Cols. 6, 7 and 8 the major axis, the minor axis and the position angle of each component. Finally, Cols. 9 and 10 give the peak intensity and the first level contour in case an image of the source is shown in Fig. 2

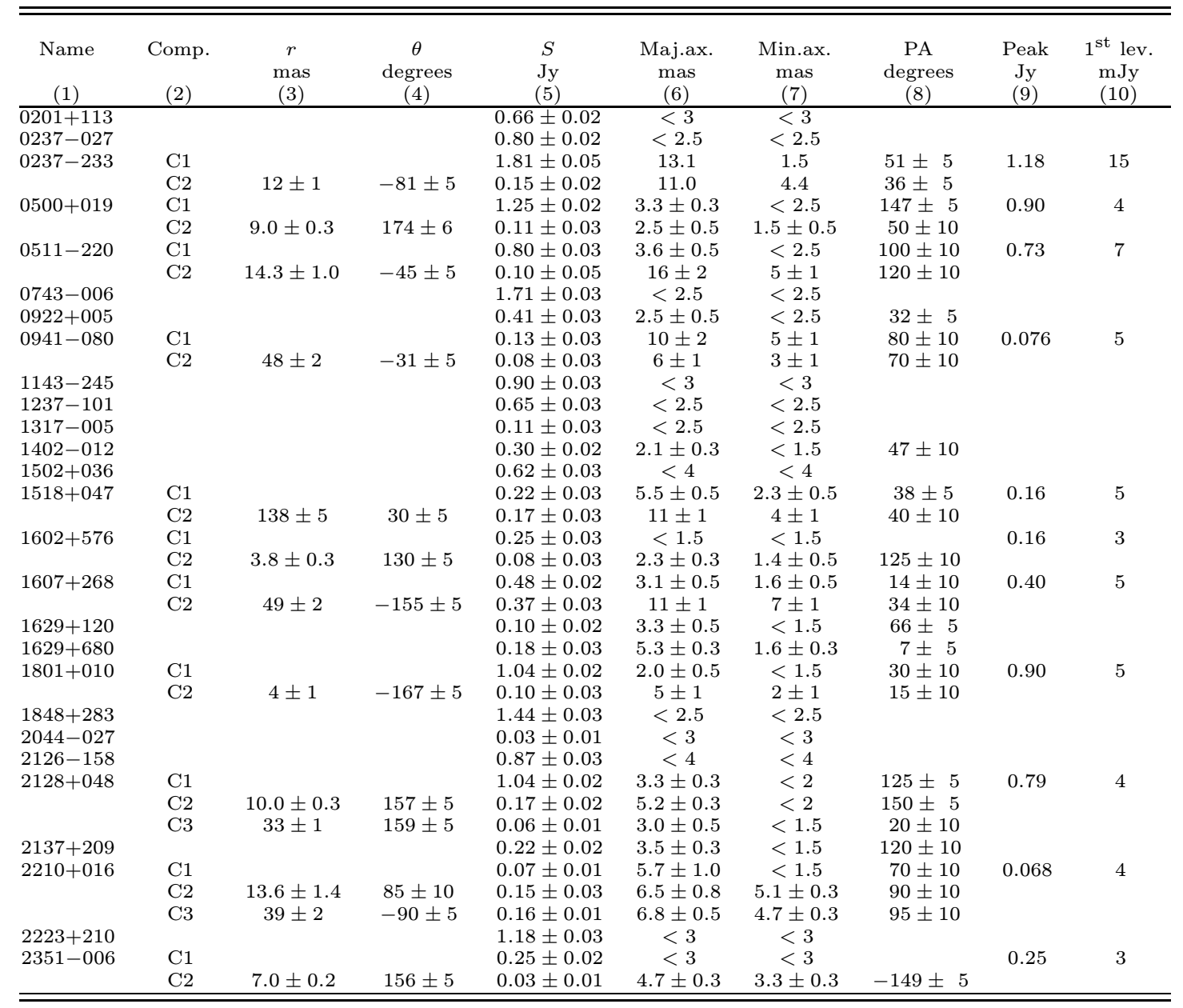

$\left.F_{X} \sim 60 \%\right)$. A comparison with a global VLBI image at $6 \mathrm{~cm}$ by Stanghellini et al. (1997) provides a confirmation of the forementioned structure, although it is not clear which component can be considered the source core. The most likely candidate is component $\mathrm{C} 1$, which is the most compact one.

$2223+210[Q, z=1.959]$

Aldcroft et al. (1994) detected a number of absorption features at $z \simeq 1.44$ and $z \simeq 1.90$ in the optical spectrum. There is also a positive detection in the X-rays (Wilkes et al. 1994).

The integrated radio spectrum reveals possible variability of this quasar (as can be derived from a comparison between the $2.7 \mathrm{GHz}$ flux densities reported in Kühr et al.
1981 and in the new Parkes catalogue). It is difficult to determine the optically thin spectral index, since we would need simultaneous measurements at the various frequencies, however it might be consistent with a CSS type, with a possible turnover at about $400 \mathrm{MHz}$.

The VLA image at $5 \mathrm{GHz}$ by Barthel et al. (1988) shows a secondary weak component at about $1^{\prime \prime}$ in PA $30^{\circ}$, responsible for a small fraction of the total flux density, and a deeper image by Lonsdale et al. (1993) shows also a third component at about $5^{\prime \prime}$ on the opposite side, although it might also be an unrelated object.

The mas structure is dominated by a single component, which is barely resolved at $S$ band. The total flux density in the $X$ band exceeds the measurement reported in the Parkes catalogue by about $30 \%$. On the other hand, the $S$ band image accounts for about half of the total flux density. 
Table 4. Source models at $S$ band $(2.3 \mathrm{GHz})$. Column 1 gives the source name, Col. 2 shows the component name derived from the images of Fig. 2; Cols. 3 and 4 show the radial distance and position angle of the various component derived with respect to component C1. Column 5 gives the flux density; Cols. 6, 7 and 8 the major axis, the minor axis and the position angle of each component. Finally, Cols. 9 and 10 give the peak intensity and the first level contour in case an image of the source is shown in Fig. 2

\begin{tabular}{|c|c|c|c|c|c|c|c|c|c|}
\hline $\begin{array}{c}\text { Name } \\
\text { (1) }\end{array}$ & $\begin{array}{c}\text { Comp. } \\
\text { (2) }\end{array}$ & $\begin{array}{c}r \\
\operatorname{mas} \\
(3)\end{array}$ & $\begin{array}{c}\theta \\
\text { degrees } \\
(4)\end{array}$ & $\begin{array}{c}S \\
\text { Jy } \\
(5)\end{array}$ & $\begin{array}{c}\text { Maj.ax. } \\
\text { mas } \\
(6)\end{array}$ & $\begin{array}{c}\text { Min.ax. } \\
\text { mas } \\
(7)\end{array}$ & $\begin{array}{c}\text { PA } \\
\text { degrees } \\
(8)\end{array}$ & $\begin{array}{c}\text { Peak } \\
\text { Jy } \\
(9)\end{array}$ & $\begin{array}{c}1^{\text {st }} \text { lev. } \\
\text { mJy } \\
(10)\end{array}$ \\
\hline $0201+113$ & & & & $0.57 \pm 0.02$ & $<7$ & $<7$ & & & \\
\hline $0237-027$ & & & & $0.27 \pm 0.02$ & $9.7 \pm 1.0$ & $<6$ & $111 \pm 10$ & & \\
\hline $0237-233$ & & & & $3.20 \pm 0.05$ & $<5$ & $<5$ & & & \\
\hline $0500+019$ & & & & $1.58 \pm 0.05$ & $10.2 \pm 0.6$ & $<8$ & $157 \pm 10$ & & \\
\hline $0511-220$ & & & & $0.33 \pm 0.02$ & $<6$ & $<6$ & & & \\
\hline $0743-006$ & & & & $0.70 \pm 0.02$ & $<8$ & $<8$ & & & \\
\hline $0922+005$ & & & & $0.43 \pm 0.02$ & $7 \pm 1$ & $<6$ & $46 \pm 10$ & & \\
\hline \multirow{2}{*}{$0941-080$} & $\mathrm{C} 1$ & & & $0.46 \pm 0.03$ & $18 \pm 1$ & $11 \pm 1$ & $53 \pm 10$ & 0.38 & 6 \\
\hline & $\mathrm{C} 2$ & $52 \pm 2$ & $135 \pm 5$ & $0.49 \pm 0.02$ & $16 \pm 1$ & $11 \pm 1$ & $123 \pm 10$ & & \\
\hline $1143-245$ & & & & $1.09 \pm 0.02$ & $11 \pm 1$ & $<13$ & $114 \pm 10$ & & \\
\hline $1237-101$ & & & & $0.78 \pm 0.03$ & $11 \pm 1$ & $<8$ & $132 \pm 10$ & & \\
\hline $1317-005$ & & & & $0.18 \pm 0.02$ & $<7$ & $<7$ & & & \\
\hline $1402-012$ & & & & $0.48 \pm 0.02$ & $<6$ & $<6$ & & & \\
\hline $1502+036$ & & & & $0.50 \pm 0.02$ & $4.3 \pm 1.0$ & $<8$ & $119 \pm 10$ & & \\
\hline \multirow{2}{*}{$1518+047$} & $\mathrm{C} 1$ & & & $0.70 \pm 0.05$ & $12.6 \pm 1.0$ & $4.0 \pm 1.0$ & $46 \pm 5$ & 0.72 & 6 \\
\hline & C2 & $138 \pm 3$ & $27 \pm 5$ & $1.00 \pm 0.03$ & $15.8 \pm 0.5$ & $4.7 \pm 1.0$ & $37 \pm 5$ & & \\
\hline \multirow[t]{3}{*}{$1602+576$} & $\mathrm{C} 1+\mathrm{C} 2$ & & & $0.53 \pm 0.02$ & $<5$ & $<5$ & & 0.51 & 6 \\
\hline & C3 & $72 \pm 2$ & $127 \pm 5$ & $0.015 \pm 0.01$ & $10 \pm 1$ & $5 \pm 1$ & $92 \pm 5$ & & \\
\hline & $\mathrm{C} 4$ & $120 \pm 2$ & $149 \pm 5$ & $0.04 \pm 0.01$ & $14 \pm 2$ & $4 \pm 1$ & $90 \pm 5$ & & \\
\hline \multirow[t]{2}{*}{$1607+268$} & $\mathrm{C} 1$ & & & $1.29 \pm 0.02$ & $8 \pm 2$ & $<3$ & $50 \pm 10$ & 1.18 & 6 \\
\hline & $\mathrm{C} 2$ & $47 \pm 1$ & $-155 \pm 5$ & $1.09 \pm 0.02$ & $11 \pm 1$ & $4 \pm 2$ & $22 \pm 10$ & & \\
\hline \multirow[t]{2}{*}{$1629+120$} & $\mathrm{C} 1$ & & & $0.10 \pm 0.02$ & $<10$ & $<10$ & & 0.36 & 5 \\
\hline & $\mathrm{C} 2$ & $1140 \pm 10$ & $101 \pm 5$ & $0.21 \pm 0.02$ & $28 \pm 2$ & $13 \pm 2$ & $146 \pm 10$ & & \\
\hline \multirow[t]{2}{*}{$1629+680$} & $\mathrm{C} 1$ & & & $0.44 \pm 0.02$ & $56 \pm 1$ & $<5$ & $25 \pm 5$ & 0.41 & 7 \\
\hline & $\mathrm{C} 2$ & $23 \pm 2$ & $180 \pm 15$ & $0.29 \pm 0.02$ & $41 \pm 2$ & $8 \pm 3$ & $2 \pm 5$ & & \\
\hline $1801+010$ & & & & $1.60 \pm 0.05$ & $19 \pm 1$ & $10 \pm 1$ & $163 \pm 10$ & & \\
\hline $1848+283$ & & & & $0.25 \pm 0.01$ & $<7$ & $<7$ & & & \\
\hline $2044-027$ & $\mathrm{C} 1$ & & & $0.19 \pm 0.05$ & $25 \pm 5$ & $17 \pm 5$ & $85 \pm 10$ & 0.14 & 6 \\
\hline & $\mathrm{C} 2$ & $50 \pm 10$ & $130 \pm 15$ & $0.16 \pm 0.05$ & $65 \pm 10$ & $15 \pm 5$ & $110 \pm 10$ & & \\
\hline $2126-158$ & & & & $0.56 \pm 0.02$ & $<10$ & $<10$ & & & \\
\hline \multirow[t]{3}{*}{$2128+048$} & $\mathrm{C} 1+\mathrm{C} 2$ & & & $1.85 \pm 0.03$ & $310 \pm 1$ & $<7$ & $150 \pm 10$ & 1.55 & 6 \\
\hline & C3 & $31 \pm 1$ & $160 \pm 5$ & $0.35 \pm 0.02$ & $18 \pm 1$ & $9 \pm 1$ & $165 \pm 10$ & & \\
\hline & $\mathrm{C} 4$ & $51 \pm 2$ & $-175 \pm 5$ & $0.09 \pm 0.07$ & $22 \pm 2$ & $8 \pm 2$ & $36 \pm 10$ & & \\
\hline \multirow[t]{2}{*}{$2137+209$} & $\mathrm{C} 1$ & & & $0.47 \pm 0.02$ & $4.9 \pm 0.5$ & $<7$ & $130 \pm 20$ & 0.42 & 6 \\
\hline & $\mathrm{C} 2$ & $47.5 \pm 1.0$ & $-125 \pm 5$ & $0.16 \pm 0.01$ & $31 \pm 2$ & $25 \pm 1$ & $70 \pm 10$ & & \\
\hline \multirow[t]{3}{*}{$2210+016$} & $\mathrm{C} 1+\mathrm{C} 2$ & & & $0.44 \pm 0.03$ & $20 \pm 2$ & $6 \pm 2$ & $83 \pm 10$ & 0.36 & 6 \\
\hline & C3 & $43 \pm 3$ & $-90 \pm 5$ & $0.45 \pm 0.02$ & $13 \pm 1$ & $7 \pm 1$ & $72 \pm 10$ & & \\
\hline & $\mathrm{C} 4$ & $35 \pm 5$ & $120 \pm 5$ & $0.40 \pm 0.05$ & $17 \pm 2$ & $17 \pm 2$ & & & \\
\hline $2223+210$ & & & & $0.79 \pm 0.02$ & $12 \pm 1$ & $4 \pm 1$ & $70 \pm 5$ & & \\
\hline $2351-006$ & & & & $0.29 \pm 0.01$ & $5 \pm 1$ & $<7$ & $170 \pm 10$ & & \\
\hline
\end{tabular}

$2351-006[Q, z=0.46]$

This object has been identified with a quasar at relatively low redshift (Hewitt et al. 1995). The EVN images at both frequencies are dominated by a pointlike component accounting for about two thirds of the total flux density. The information available in the literature is rather sparse; consequently, the integrated radio spectrum has very few points and does not allow a proper classification.

\subsection{Session 2}

As mentioned in Sect. 2, in May 1994 we observed 4 objects, spending about 2 hrs on each source. This allowed a better filled $u v$-plane and therefore a higher image fidelity.
$1402-012[Q, z=2.518]$

This high redshift quasar has been reported to be optically variable (Pica et al. 1988), and to have a number of absorption features in the optical spectrum (Junkkarinen et al. 1991). Moreover is has also been detected by the Einstein satellite in the X-rays (Wilkes et al. 1994).

The radio flux densities collected from the literature do not allow a complete determination of the radio spectrum. The mas radio emission is dominated by a single component at both frequencies, barely resolved at $X$ band, where the whole flux density is accounted for. On the other hand 
Table 5. Source parameters. For each source, we used the most convenient image (either $X$ or $S$ ), according to the component size. For the source $2210+106$ without a measured redshift, a value of $z=1$ has been used. Column 1 gives the source name, Col. 2 the component name; Cols. 3 and 4 provide the minimum energy density and the magnetic field computed assuming equipartition conditions

\begin{tabular}{|c|c|c|c|c|c|c|c|}
\hline $\begin{array}{c}\text { Name } \\
\text { (1) }\end{array}$ & $\begin{array}{l}\text { Comp. } \\
\text { (2) }\end{array}$ & $\begin{array}{c}u_{\min } \\
10^{-5} \mathrm{erg} / \mathrm{cm}^{3} \\
(3)\end{array}$ & $\begin{array}{c}H_{\mathrm{eq}} \\
\text { mGauss } \\
(4)\end{array}$ & $\begin{array}{c}\text { Name } \\
(1)\end{array}$ & $\begin{array}{l}\text { Comp. } \\
(2)\end{array}$ & $\begin{array}{c}u_{\min } \\
10^{-5} \mathrm{erg} / \mathrm{cm}^{3} \\
(3)\end{array}$ & $\begin{array}{c}H_{\mathrm{eq}} \\
\mathrm{mGauss} \\
(4)\end{array}$ \\
\hline $0201+113$ & & $>41$ & $>67$ & $1629+120$ & $\mathrm{C} 1$ & $>7.2$ & $>28$ \\
\hline $0237-027$ & & $>10$ & $>33$ & & $\mathrm{C} 2$ & 0.15 & 4.1 \\
\hline \multirow[t]{2}{*}{$0237-233$} & $\mathrm{C} 1$ & & & $1629+680$ & $\mathrm{C} 1$ & 10 & 33 \\
\hline & $\mathrm{C} 2$ & & & & $\mathrm{C} 2$ & 0.34 & 6.0 \\
\hline \multirow[t]{2}{*}{$0500+019$} & $\mathrm{C} 1$ & $>5.9$ & $>25$ & $1801+010$ & $\mathrm{C} 1$ & $>32$ & 59 \\
\hline & $\mathrm{C} 2$ & 2.9 & 18 & & $\mathrm{C} 2$ & 3.0 & 18 \\
\hline \multirow[t]{2}{*}{$0511-220$} & $\mathrm{C} 1$ & $>8.9$ & $>31$ & $1848+283$ & & $>47$ & $>71$ \\
\hline & $\mathrm{C} 2$ & 0.42 & 6.7 & $2044-027$ & & $>0.95$ & $>10$ \\
\hline $0743-006$ & & $>14$ & $>39$ & & $\mathrm{C} 2$ & 0.03 & 1.6 \\
\hline $0922+005$ & & $>12$ & $>36$ & $2126-158$ & & $>25$ & $>51$ \\
\hline \multirow[t]{2}{*}{$0941-080$} & $\mathrm{C} 1$ & 0.02 & 1.6 & $2128+048$ & C1 & $>9.9$ & $>33$ \\
\hline & $\mathrm{C} 2$ & 0.03 & 1.7 & & $\mathrm{C} 2$ & $>2.4$ & $>16$ \\
\hline $1143-245$ & & $>17$ & $>43$ & & C3 & $>2.7$ & $>17$ \\
\hline $1237-101$ & & $>6.2$ & $>26$ & & $\mathrm{C} 4$ & 0.08 & 3.0 \\
\hline $1317-005$ & & $>2.6$ & $>17$ & $2137+209$ & $\mathrm{C} 1$ & $>8.7$ & $>31$ \\
\hline $1402-012$ & & $>33$ & $>60$ & & $\mathrm{C} 2$ & 0.06 & 2.5 \\
\hline $1502+036$ & & $>1.9$ & $>14$ & $2210+016$ & $\mathrm{C} 1$ & $>1.7$ & $>14$ \\
\hline \multirow[t]{2}{*}{$1518+047$} & $\mathrm{C} 1$ & 3.2 & 19 & & $\mathrm{C} 2$ & 0.83 & 9.5 \\
\hline & $\mathrm{C} 2$ & 0.94 & 10 & & C3 & 0.89 & 9.8 \\
\hline \multirow[t]{4}{*}{$1602+576$} & $\mathrm{C} 1$ & $>50$ & $>73$ & & $\mathrm{C} 4$ & 0.12 & 3.7 \\
\hline & $\mathrm{C} 2$ & 19 & 45 & $2223+210$ & & $>20$ & $>46$ \\
\hline & C3 & 0.63 & 8.3 & $2351-006$ & $\mathrm{C} 1$ & $>1.9$ & $>14$ \\
\hline & $\mathrm{C} 4$ & 0.37 & 6.3 & & $\mathrm{C} 2$ & 0.36 & 6.2 \\
\hline \multirow[t]{2}{*}{$1607+268$} & $\mathrm{C} 1$ & $>4.7$ & $>23$ & & & & \\
\hline & $\mathrm{C} 2$ & 0.39 & 6.5 & & & & \\
\hline
\end{tabular}

at $S$ band only about $75 \%$ of the total flux is present in the image (not shown).

$1602+576[Q, z=2.85](4 \mathrm{C}+57.27)$

The $S$ band image shows two asymmetric components separated by about 120 mas. The flux density ratio is about $25: 1$. The $X$ band image shows a single component likely to be identified with the brighter one at $X$ band. Both images account for the whole flux density of the radio source at each frequency respectively. The integrated spectrum is poorly defined due to the lack of measurements in the literature.

$1629+680[Q, z=2.475](4 \mathrm{C}+68.18)$

This quasar shows an absorption feature in the optical spectrum (Junkkarinen et al. 1991). The radio spectrum is straight and rather steep. The VLBI images show a well resolved jet-like structure. The size of the radio emission is $\sim 50$ mas at $2.3 \mathrm{GHz}$, with indications for a possible $90^{\circ}$ bend. The structure imaged at $8.3 \mathrm{GHz}$ is much shorter due to the lower surface brightness and less sensitivity to the extended structure. This source has been found to be unresolved (angular size smaller than 0.25 arcsec) with MERLIN at $408 \mathrm{MHz}$ and with the VLA at $5 \mathrm{GHz}$ by Reid et al. (1995)

$1801+010[Q, z=1.522]$

This source was taken from Barthel et al. (1988), and has a rather flat spectrum which steepens at about $5 \mathrm{GHz}$. Some variability can also be inferred from literature data. Our VLBI images are dominated by a pointlike component. The spectral index between $S$ and $X$ bands is 0.34 . Simard-Normandin et al. (1981) found $13 \%$ fractional polarization at $L$ band. 


\section{Discussion and summary}

Table 3 and Table 4 show the parameters derived from the present data. When possible the modelfitting was done independently of the image plane by means of the AIPS task JMFIT and also using the visibilities using the task modelfit of the Caltech-Difmap package. Generally the solutions found were in good agreement, and we used the differences in the results provided by the two methods as an estimate of the errors on the parameters reported in the tables. The component labelling is reported on the images for those sources were multiple components have been fitted.

In Table 5 we summarize the physical parameters of the sources as derived from the assumption of equipartition conditions within the radio emitting region. We further assumed $\delta=2.6$ as index of the energy distribution of the relativistic electrons (corresponding to an optically thin spectral index of 0.8 , in a region not affected by synchrotron ageing), a filling factor $\Phi=1$ and particle energy equally shared between electrons and protons $(k=1)$, integrating the radio spectrum between $10 \mathrm{MHz}$ and $100 \mathrm{GHz}$, using the relationship by Miley (1980). When unknown, we arbitrarily used $z=1$. We did not apply the "k-correction" to take into account the values in the rest frame, since the instrinsic values are not discussed. Finally the parameters are derived at the most convenient frequency (either 2.3 or $8.3 \mathrm{GHz}$ ) where the radio emitting component is better described (the former for resolved components, the latter for the unresolved regions). Generally the equipartition magnetic field is of the order of $10^{-2}-10^{-3}$ Gauss, while the minimum energy density is about $10^{-4}-10^{-5} \mathrm{erg} \mathrm{cm}^{-3}$. These figures are higher than those found in CSS sources, and are similar to those derived for the GPS sources with the highest turnover frequencies. This is partly due to the compactness of the majority of the components detected here; in fact the present data are more favorable to the detection of high brightness compact regions.

All the six galaxies in the sample show some structure, which can always be interpreted in terms of a double (often asymmetric) or a triple source. Quasars instead are mostly found to be represented by a single component and the incidence of significant VLBI structure (core-jet or double) is limited to 8 objects out of 22 . This might also be due to the low dynamic range of our images which prevents the detection of low surface brightness regions in objects with a very bright component or very asymmetric radio emission. In general the radio structures we observe are consistent with those seen by Stanghellini et al. (1997) for their sample of powerful GPS sources.

Most of the sources presented here can be considered good GPS candidates, since the properties we were able to study are those typical of the CSS/GPS class. Flux density variability has been inferred from literature data for a few candidates (all quasars), but the increase/decrease was never enough to exclude the candidate. We note that the optically thin spectral index is flatter than 0.5 in $0922+$ 005, while a flattening at $\mathrm{mm}$ wavelengths is likely to occur in $0201+113$ and $0237-027$. On the other hand, a number of sources have ill defined radio spectra due to the lack of data; in particular, further multifrequency flux density measurements would be required for $0237-027,0941+$ $261,1402-012,1502+036,1602+576,1801+010$ and $2351-006$.

Acknowledgements. We thank the European VLBI Network and the personnel at the geodetic stations of Matera and Wettzell. This research has made use of the NASA/IPAC Extragalactic Database (NED), which is operated by the Jet Propulsion Laboratory, California Institute of Technology, under contract with the National Aeronautics and Space Administration. DD and MB acknowledges the Commission of the European Union for the award of a Fellowship under contract ERBCHBGCT920212. DD acknowledges the Joint Institute for VLBI in Europe (JIVE) and the National Foundation for Research in Astronomy (Dwingeloo, NL) and MB acknowledges the Nuffield Radio Astronomy Laboratories (Jodrell Bank, UK) for hospitality during the fellowship. DD and MB acknowledge the Italian Space Agency (ASI) for support under contract CNR/ASI ARS 96-13. We wish to thank Richard Schilizzi for the encouragement and the referee, Chris O'Dea, for insightful comments on the manuscript.

\section{References}

Aldcroft T.L., Bechtold J., Elvis M., 1994, ApJS 93, 1

Barthel P.D., Miley G.K., Schilizzi R.T., Lonsdale C.J., 1988, A\&AS 73, 515

Baum S.A., O'Dea C.P., de Bruyn A.G., Murphy D.W., 1990, A\&A 232, 19

Becker R.H., White R.L., Edwards A.L., 1991, ApJS 75, 1

Bechtold J., Elvis M., Fiore F., et al., 1994, AJ 108, 374

van Breugel W.J.M, Miley G.K., Heckman T.M., 1984, AJ 89, 5

de Bruyn A.G., O’Dea C.P., Baum S.A., 1996, A\&A 305, 450

Carvalho J.C., 1985, MNRAS 215, 463

Dallacasa D., Stanghellini C., 1991, in Proc of "Compact SteepSpectrum and GHz-Peaked Spectrum Radio Sources", Fanti C., Fanti R., O'Dea C.P., Schilizzi R.T. (eds.), Consiglio Nazionale delle Ricerche - Istituto di Radioastronomia, Bologna, p. 224

Dallacasa D., Fanti C., Fanti R., Schilizzi R.T., Spencer R.E., 1995, A\&A 295, 27

Elvis M. Wilkes B.J., McDowell J.C., et al., 1994, ApJS 95, 1

Fanti R., Fanti C., Schilizzi R.T., et al., 1990, A\&A 231, 333

Fanti C., Fanti R., Dallacasa D., et al., 1995, A\&A 302, 317

Fugmann W., Meisenheimer K., 1988, A\&AS 76, 145

Ghosh T., Rao A.P., 1992, A\&A 264, 203

Ghosh T., Gopal-Krishna Rao A.P., 1994, A\&AS 106, 29

Griffith M.R., Wright A.E., Burke B.F., Ekers R.D., 1995, ApJS 97, 347

Hewitt A., Burbidge G., 1989, ApJS 69, 1

Hewitt P.C., Foltz C.B., Chaffee F.H., 1995, AJ 109, 1498

Hodges M.W., Mutel R.L., Phillips R.B., 1984, AJ 89, 1327

Junkkarinen V., Hewitt A., Burbidge G., 1991, ApJS 77, 203 
Kühr H., Witzel A., Pauliny-Toth I.I.K., Nauber U., 1981, A\&AS 45, 367

Lonsdale C.J., Barthel P.D., Miley G.K., 1993, ApJS 87, 63

Miley G.K., 1980, ARA\&A 18, 165

Morabito D.D., Niell A.E., Preston R.A., et al., 1986, AJ 91, 1038

Mutel R.L., Hodges M.W., Phillips R.B., 1985, ApJ 290, 86

Mutel R.L., Hodges M.W., 1986, ApJ 307, 472

Mutel R.L., Phillips R.B., 1988, Proc. IAU Symp. 129, Reid M.J., Moran J.M. (eds.), p. 73

Neff S.G., Hutchings J.B., 1990, AJ 100, 1441

O'Dea C.P., Baum S.A., Stanghellini C., et al., 1990, A\&AS 84, 549

O’Dea C.P., Baum S.A., Stanghellini C., 1991, ApJ 380, 66

O'Dea C.P., Stanghellini C., Baum S.A., Charlot S., 1996, ApJ 470, 806

O'Dea C.P., Baum S.A., 1997, AJ 113, 1480

Owen F.N., Spangler S.R., Cotton W.D., 1980, AJ 85, 351

Perley R.A., 1982, AJ 87, 859

Phillips R.B., Mutel R.L., 1982, A\&A 106, 21

Pica A.J., Smith A.G., Webb J.R., et al., 1988, AJ 96, 1215

Preston R.A., Jauncey D.L., Meier D.L., et al., 1989, AJ 98, 1

Price R., Gower A.C., Hutchings J.B., et al., 1993, ApJS 86, 365

Readhead A.C.S., Taylor G.B., Pearson T.J., Wilkinson P.N., 1996, ApJ 460, 634

Reid A., Shone D.L., Akujor C.E., et al., 1995, A\&AS 110, 213

Rusk R., 1988, PhD Thesis, Univ. of Toronto
Simard-Normandin M., Kronberg P.P., Neidhofer J., 1981, A\&AS 43, 19

Snellen I.A.G., Zhang M., Schilizzi R.T., et al., 1995, A\&A 300, 359

Spangler S.R., Mutel R.L., Benson J.M., 1983, ApJ 271, 44

Spencer R.E., McDowell J.C., Charlesworth M., 1989, MNRAS 240,657

Stanghellini C., Baum S.A., O’Dea C.P., Morris G.B., 1990, A\&A 233, 379

Stanghellini C., O'Dea C.P., Baum S.A., Laurikainen E., 1993, ApJS 88, 1

Stanghellini C., O'Dea C.P., Baum S.A., et al., 1997, A\&A (in press)

Steppe H., Liechti S., Mauersberger R., et al., 1992, A\&AS 96, 411

Steppe H., Jeyakumar S., Saikia D.J., Salter C.J., 1995, A\&AS 113, 409

Stickel M., Kühr H., 1996, A\&AS 115, 11

de Vries W.H., Barthel P.D., Hes R., 1995, A\&AS 114, 259

Waltman E.B., Fiedler R.L., Johnston K.J., et al., 1991, ApJS 77,379

White R.L., Kinney A.L., Becker R.H., 1993, APJ 407, 456

Wilkes B.J., Tananbaum H., Worral D.M., et al., 1994, ApJS 92,53

Wills B.J., Wills D., Evans N.J., et al., 1992, ApJ 398, 454

Wills D., 1979, ApJS 39, 291

Wiren S., Valtaoja E., Terasranta H, Kotilainen J., 1992, AJ 104, 1009 